EUROPEAN COOPERATION

IN THE FIELD OF SCIENTIFIC

AND TECHNICAL RESEARCH
IC1004 TD(14)11017

Krakov, Poland

September 24-26, 2014

\begin{tabular}{ll}
\hline & EURO-COST \\
\hline SOURCE: & Eurecom \\
& IFSTTAR \\
& SNCF
\end{tabular}

Broadband Wireless Channel Measurements for High Speed Trains

Florian Kaltenberger*, Auguste Byiringiro*, George Arvanitakis*, Riadh

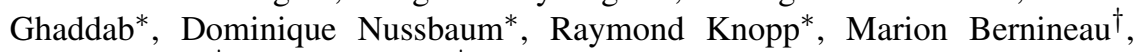
Yann Cocheril ${ }^{\dagger}$, Henri Philippe ${ }^{\ddagger}$

${ }^{*}$ EURECOM, Sophia Antipolis, France

$\dagger$ IFSTTAR

$\ddagger$ SNCF

Phone: +33(0)493008100

Fax: $\quad+33(0) 493008200$

Email: florian.kaltenberger@eurecom.fr 


\title{
Broadband Wireless Channel Measurements for High Speed Trains
}

\author{
Florian Kaltenberger*, Auguste Byiringiro*, George Arvanitakis*, Riadh Ghaddab*, \\ Dominique Nussbaum*, Raymond Knopp*, Marion Bernineau ${ }^{\dagger}$, Yann Cocheril ${ }^{\dagger}$, \\ Henri Philippe ${ }^{\ddagger}$ \\ *EURECOM, Sophia Antipolis, France \\ ${ }^{\dagger}$ IFSTTAR \\ ${ }^{\ddagger} \mathrm{SNCF}$
}

\begin{abstract}
We describe a channel sounding measurement campaign for cellular broadband wireless communications with high speed trains that was carried out in the context of the project CORRIDOR. The campaign combines MIMO and carrier aggregation to achieve very high throughputs. We compare two different scenarios, the first one reflects a cellular deployment, where the base station is about $1 \mathrm{~km}$ away from the railway line. The second scenario corresponds to a railway deployed network, where the base station is located directly next the the railway line.

We present the general parameters of the measurement campaign and some preliminary results of Power Delay Profiles and Doppler Spectra and their evolution over time.
\end{abstract}

Index Terms-MIMO, Carrier Aggregation, Channel Sounding, High-speed train

\section{INTRODUCTION}

Broadband wireless communications has become an ubiquitous commodity. However, there are still certain scenarios where this commodity is not available or only available in poor quality. This is certainly true for high speed trains traveling at $300 \mathrm{~km} / \mathrm{h}$ or more.

While the latest broadband communication standard, LTE, has been designed for datarates of $150 \mathrm{Mbps}$ and speeds of up to $500 \mathrm{~km} / \mathrm{h}$, the practical achievable rates are significantly lower. A recent experiment carried out by Ericsson showed that the maximum achievable datarate was $19 \mathrm{Mpbs}$ on a jet plane flying at $700 \mathrm{~km} / \mathrm{h}^{1}$.

Two main technologies exist to increase datarates: using multiple antennas to form a multiple-input multipleoutput (MIMO) system, and using more spectrum by means of carrier aggregation (CA). While MIMO has been already included in the first versions of the LTE standard (Rel. 8), CA has only been introduced with LTE-Advanced (Rel.10).

To design efficient algorithms that can exploit these two technologies in high-speed conditions it is of utmost importance to have a good understanding of the channel conditions. While some measurements exist for

${ }^{1} \mathrm{http}: / /$ www.ericsson.com/news/121101-ericsson-tests-lte-inextreme-conditions_244159017_c

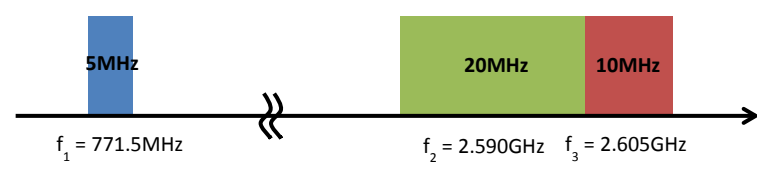

Fig. 1. The sounding signal is composed of 3 component carriers, each of which uses 4 transmit antennas

SISO channels [1], [2], there are no reports of MIMO measurements in high-speed trains. There are however a series of MIMO measurements (using a switched array) available for vehicular communications at speeds of up to $130 \mathrm{~km} / \mathrm{h}[3]$.

To the best of the author's knowledge the measurements presented in this paper are the first measurements that combine MIMO with carrier aggregation at very high speeds of up to $300 \mathrm{~km} / \mathrm{h}$. Moreover, our MIMO measurement system does not use a switched array, but records channels in parallel.

The rest of the paper is organized as follows. We first present the measurement equipment and methodology in Section II, followed by a description of the measurement scenarios in Section III. We present the post-processing in Section IV and the results in Section V. Finally we give conclusions in Section VI.

\section{MEAsurement EQuiPMEnT AND METHODOLOGY}

\section{A. Sounding Signal}

The sounding signal was designed based on constraints given by the hardware (number of antennas) and the obtained licenses for spectrum user (number of carriers). The final design uses 3 carriers as depicted in Figure 1, each of which uses four transmit antennas.

Each carrier is using an OFDM signal, whose parameters are similar to those of the LTE standard. Table I summarizes the signal parameters.

The signal is framed to $10 \mathrm{~ms}$, or 120 OFDM symbols. The first symbol of each frame contains the LTE primary 


\begin{tabular}{|c|c|c|c|}
\hline & $5 \mathrm{MHz}$ & $10 \mathrm{MHz}$ & $20 \mathrm{MHz}$ \\
\hline \hline Sampling rate (Msps) & 7.68 & 15.36 & 30.72 \\
\hline OFDM symbol duration & \multicolumn{3}{|c|}{$66 \mu \mathrm{s}$} \\
\hline Cyclic prefix length & \multicolumn{3}{|c|}{$16 \mu \mathrm{s}$} \\
\hline OFDM symbol size & 512 & 1024 & 2048 \\
\hline Useful OFDM carriers & 300 & 600 & 1200 \\
\hline
\end{tabular}

TABLE I

SOUNDING SIGNAL PARAMETERS

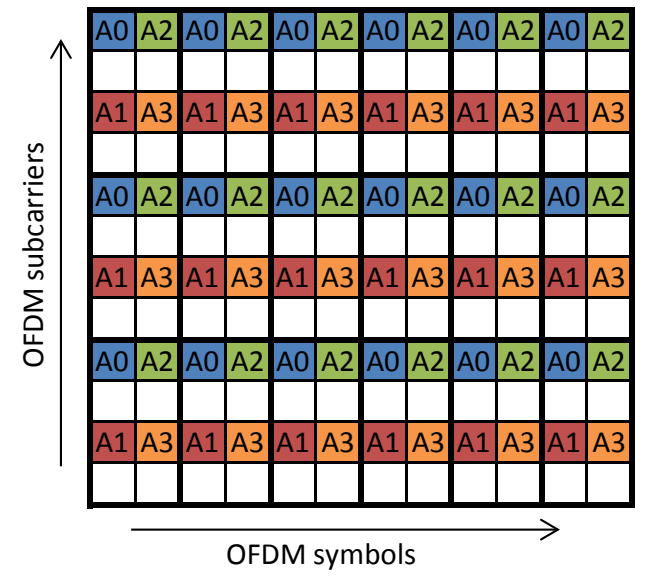

Fig. 2. Allocation of resource elements (RE) to antennas. Empty REs are unused to reduce inter carrier interference (ICI).

synchronization sequence (PSS) and the rest of the signal is filled with OFDM modulated random QPSK symbols. In order to minimize inter carrier interference (ICI) in high mobility scenarios, we only use ever second subcarrier. To obtain individual channel estimates from the different transmit antennas, we use an orthogonal pilot pattern as depicted in Figure 2.

\section{B. Measurement Equipment}

The basis for both transmitter and receiver of the channel sounder is the Eurecom ExpressMIMO2 software defined radio card (see Figure 3), which are part of the OpenAirInterface platform ${ }^{2}$. The card features four independent RF chains that allow to receive and transmit on carrier frequencies from $300 \mathrm{MHz}$ to $3.8 \mathrm{GHz}$. The digital signals are transfered to and from the PCI in realtime via a PCI Express interface. The sampling rate of the card can be chosen from $n \cdot 7.68 \mathrm{Msps}, n=1,2,4$, corresponding to a channelization of 5,10 , and $20 \mathrm{MHz}$. However, the total throughput of one card may not exceed the equivalent of one $20 \mathrm{MHz}$ channel due to the current throughput limitation on the PCI Express interface. Thus the following configurations are allowed: $4 \times 5 \mathrm{MHz}, 2 \times 10 \mathrm{MHz}$, or $1 \times 20 \mathrm{MHz}$.

Multiple cards can be synchronized and stacked in a PCI chassis to increase either the bandwidth or the

${ }^{2} \mathrm{http} / / /$ www.openairinterface.org

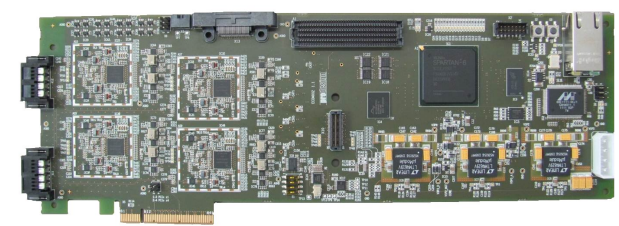

Fig. 3. Express MIMO 2 board

number of antennas. For the transmitter in this campaign we have used 7 ExpressMIMO2 cards to achieve the total aggregated bandwidth of $20+10+5=35 \mathrm{MHz}$ with 4 transmit antennas each. A schematic of the transmitter is given in Figure 4.

The output of the ExpressMIMO2 cards is limited to approximately $0 \mathrm{dBm}$, therefore additional power amplifiers have been built for bands around $800 \mathrm{MHz}$ (including TV white spaces and E-UTRA band 20) and for bands around $2.6 \mathrm{GHz}$ (E-UTRA band 7). The to achieve a total output power $40 \mathrm{dBm}$ at $800 \mathrm{MHz}$ and $+36 \mathrm{dBm}$ at 2.6 Ghz (per element).

As antennas we have used two sectorized, dual polarized HUBER+SUHNER antennas with a $17 \mathrm{dBi}$ gain (ref SPA 2500/85/17/0/DS) for the $2.6 \mathrm{GHz}$ band and two sectorized, dual polarized Kathrein antennas with a 14.2 dBi gain (ref $80010734 \mathrm{~V} 01$ ) for the $800 \mathrm{MHz}$ band (see Figure 5.

The receiver is built similarly, but it was decided to use two separate systems for the two bands. The $800 \mathrm{Mhz}$ receiver is built from one ExpressMIMO2 card, providing three $5 \mathrm{MHz}$ channels for three receive antennas. The $2.6 \mathrm{GHz}$ receiver is built from three ExpressMIMO2 cards, providing two $20 \mathrm{MHz}$ and two $10 \mathrm{MHZ}$ channels in total which are connected to two antenna ports in a similar way as the transmitter. The $2.6 \mathrm{GHz}$ receiver additionally uses external low-noise-power amplifiers with a $10 \mathrm{~dB}$ gain to improve receiver sensitivity.

The receiver antennas used are Sencity Rail Antennas from HUBER+SUHNER (see Figure 6. For the $800 \mathrm{MHz}$ band we have used two SWA $0859 / 360 / 4 / 0 / \mathrm{V}^{3}$ and one SWA 0859/360/4/0/DFRX30 ${ }^{4}$ omnidirectional antennas with $6 \mathrm{dBi}$ gain (the latter one also provides an additional antenna port for a global navigation satellite system (GNSS)). For the $2.6 \mathrm{GHz}$ band we have used two SPA $2400 / 50 / 12 / 10 / \mathrm{V}^{5}$ antennas that provide two ports each, one pointing to the front and one to the back of the train, each with $11 \mathrm{dBi}$ gain. However, for the experiments we have only used one port from each antenna that are pointing in the same direction.

\footnotetext{
${ }^{3}$ http://goo.gl/QDIRg1

${ }^{4}$ http://goo.gl/GavYgG

${ }^{5}$ http://goo.gl/xBHSv2
} 


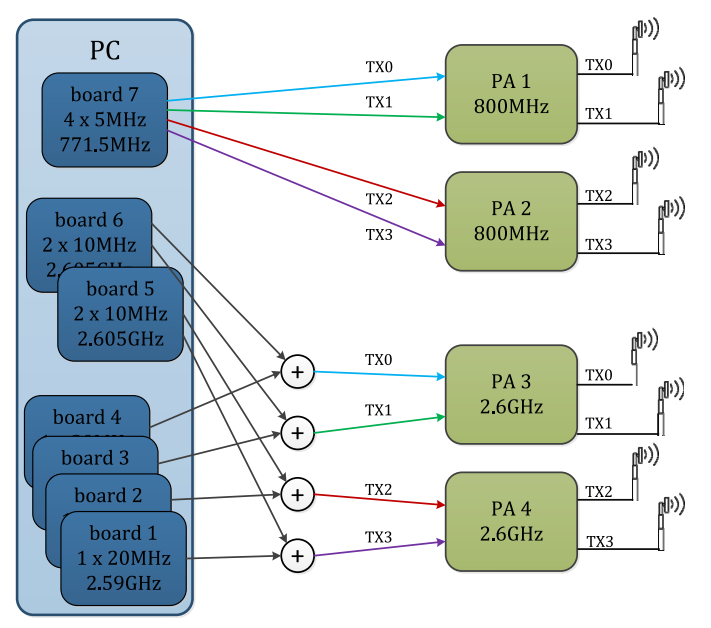

Fig. 4. Schematics of the transmitter.

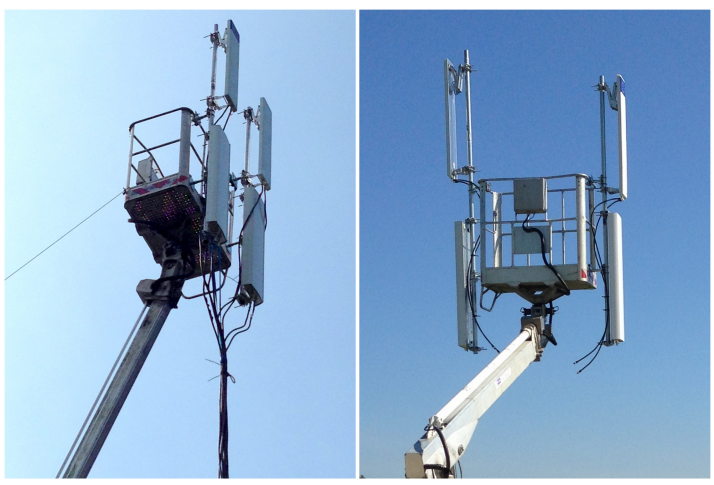

Fig. 5. Antenna setup. Left: trial 1, right: trial 2.

\section{Data acquisition}

We save the raw IQ data of all antennas in realtime. The data of the $5 \mathrm{MHz}$ channel at $771.5 \mathrm{MHz}$ is stored continuously and for the two $(10+20 \mathrm{MHz})$ channels at $2.6 \mathrm{GHz}$ we only save 1 second out of 2, due to constraints of the hard disk speed.

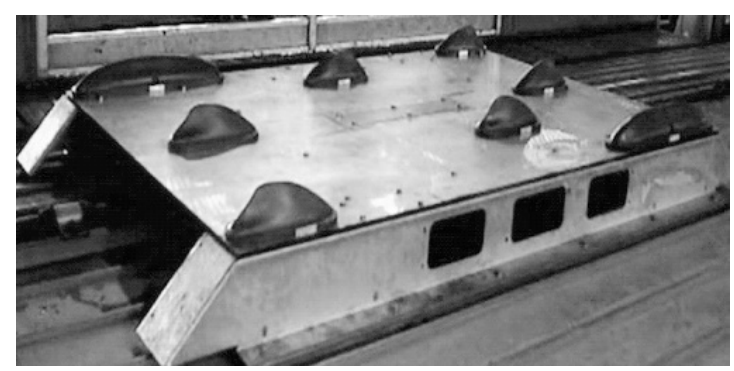

Fig. 6. Antennas on top of the IRIS 320 train.

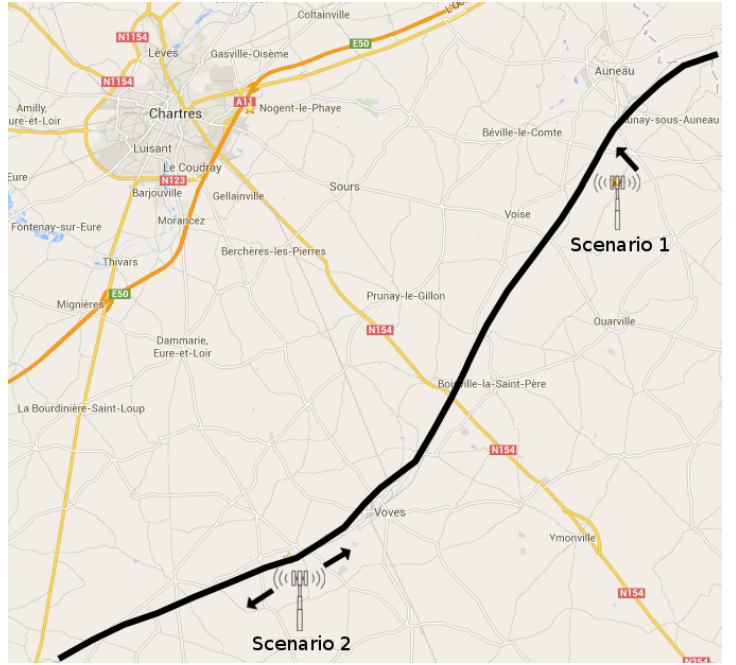

Fig. 7. Map showing the different measurement scenarios. The black line is the railway line and the arrows indicate the direction the antennas are pointing. In scenario 1 , all 4 antennas point in the same direction. In scenario $2 \mathrm{a}$, two antennas point northeast while two antennas point southwest and in scenario $2 \mathrm{~b}$ all four antennas point northeast.

\section{Measurement Scenarios AND Description}

The measurements were carried on board of the IRIS320 $\operatorname{train}^{6}$ along the railway line "LGV Atlantique" around $70 \mathrm{~km}$ southwest of Paris. The train passes the area with a speed of approximately $300 \mathrm{~km} / \mathrm{h}$. The antennas are mounted on the top of the train, approximately half way between the front and the rear.

Three scenarios were measured:

1) Scenario 1: The eNB is located $1.5 \mathrm{~km}$ away from the railway and all the TX antennas are pointing approximately perpendicular to the railway. This scenario corresponds to a cellular operator deployed network.

2) Scenario 2a: The eNB is located right next to the railway line and the half of the TX antennas pointing at one direction of the railway, and the other half are pointing at the opposite direction. This scenario corresponds to a railway operator deployed network.

3) Scenario 2b: Same as Scenario 2a, but this time all the $4 \mathrm{TX}$ antennas are oriented in the same sense.

For all scenarios the base station height is approximately $12 \mathrm{~m}$.

\section{Measurement Post Processing}

\section{A. Synchronization}

Synchronization it is the most important part of the Post processing. In OFDM systems, there exist three different problems related to synchronization: The first

\footnotetext{
${ }^{6}$ http://en.wikipedia.org/wiki/SNCF_TGV_Iris_320
} 
one is frame synchronization, which allows the receiver to determine the starting point of the received frame. The second one is the frequency synchronization, which tries to eliminate the carrier frequency offset caused by the mismatch from the radio frequency local oscillators and the Doppler shift. Finally, the last issue is the sampling clock synchronization, which manages to synchronize the sampling frequency between transmitter and receiver, because both of them work with different physical clocks.

1) Initial Timing Synchronization: To define the start of the frame we make a cross correlation between a received data and the (known) synchronization sequence (PSS) which is in the beginning of every frame. We then look for the highest peak within every frame (discarding peaks below a certain threshold) and repeat this process for several (e.g., 100) consecutive frames. We finally take the median value of the offset of the peaks within each frame.

Note that this procedure is necessary, since we have no other mean of verifying that the synchronization was achieved. In a real LTE system, after the detection of the peak of the correlator the receiver would attempt to decode the broadcast channel and thus verifying the synchronization.

2) Tracking: Due to the differences in sampling clocks between the transmitter and the receiver, the frame offset might drift over time and thus needs to be tracked and adjusted. This is done by tracking the peak of the impulse response of the estimated channel and adjusting the frame offset such that the peak is at $1 / 8$ th of the cyclic prefix. If the peak drifts further away than 5 samples, the frame offset is adjusted. This method avoids jitter of the frame offset but means that frame offset jumps a few samples.

Another possibility to compensate for the timing drift would be to apply Lanczos resampling, but this method is computational very complex and has not been applied here.

\section{B. Channel Estimation}

After synchronization we apply a standard OFDM receiver, which applies an FFT and removes the cyclic prefix. After this operation the equivalent input-output relation can be written as

$$
\mathbf{y}_{i^{\prime}, l^{\prime}}=\mathbf{H}_{i^{\prime}, l^{\prime}} \mathbf{x}_{i^{\prime}, l^{\prime}}+\mathbf{n}_{i^{\prime}, l^{\prime}}
$$

where $i^{\prime}$ denotes OFDM symbol and $l^{\prime}$ the subcarrier, $\mathbf{X}$ is the transmitted symbol vector described in Section II, $\mathbf{H}$ is the frequency domain MIMO channel matrix (MIMO transfer function) and $\mathbf{y}$ is the received symbol vector.
Since the transmitted symbols are all QPSK, we can estimate the channel matrix as

$$
\hat{\mathbf{H}}_{i^{\prime}, l^{\prime}}=\mathbf{y}_{i^{\prime}, l^{\prime}} \mathbf{x}_{i^{\prime}, l^{\prime}}^{H} .
$$

Note that due to the orthogonal structure of the transmitted pilots in $\mathbf{x}$ (cf. Figure 2), $\hat{\mathbf{H}}_{i^{\prime}, l^{\prime}}$ will be sparse. For the ease of notation and processing we thus define new indices $i=0, \ldots, N_{s}-1$ and $l=0, \ldots, N_{c}-1$ that group refer to a block of six subcarriers and two OFDM symbols respectively (these blocks are also highlighted in Figure 2). Thus $\hat{\mathbf{H}}_{i, l}$ does not contain any zero elements, $N_{s}=60$, and $N_{c}=50,100,200$ depending on the bandwith of the carrier.

For further reference we also compute the MIMO channel impulse response

$$
\hat{\mathbf{h}}_{i, k}=\mathrm{FFT}_{l}\left\{\hat{\mathbf{H}}_{i, l}\right\} .
$$

\section{Power Delay Profile Estimation}

We estimate the Power Delay Profile by averaging over all OFDM symbols $N_{s}=60$ in a frame and thus introducing a new time variable $j$ which denotes one frame $(10 \mathrm{~ms})$.

$$
\mathbf{P}_{j, k}=\frac{1}{N_{s}} \sum_{i=j N_{s}}^{(j+1) N_{s}-1}\left|\hat{\mathbf{h}}_{i, l}\right|^{2},
$$

\section{Delay-Doppler Power Spectrum Estimation}

We estimate the Delay-Doppler Power Spectrum (sometimes also called the scattering function) by taking the inverse Fourier transform of blocks of 100 frames

$$
\mathbf{S}_{t, u, k}=\frac{1}{\sqrt{100 N_{s}}} \sum_{i=100 t N_{s}}^{100(t+1) N_{s}-1} \hat{\mathbf{h}}_{i, k} e^{\frac{2 \pi j i u}{100 N_{s}}},
$$

where we have introduced the new time variable $t$ whose resolution depends on the carrier. In the case of the $5 \mathrm{MHz}$ carrier (at $800 \mathrm{MHz}$ ), it is 100 frames (1s) and in the case of the $10+20 \mathrm{MHz}$ carrier at $2.6 \mathrm{GHz}$ it is 200 frames $(2 s)$, since we only store the signal for one out of 2 seconds. This method will give us a resolution in Doppler frequency $u$ of $1 \mathrm{~Hz}$.

From $\mathbf{S}_{t, u, k}$ we can also compute the marginal Doppler profile at time $t$ by averaging over the delay time $k$

$$
\mathbf{D}_{t, u}=\frac{1}{N_{c}} \sum_{k=0}^{N_{c}-1}\left|\mathbf{S}_{t, u, k}\right|^{2},
$$

\section{Channel Characterization Results}

\section{A. Path Loss}

We estimate the path loss component as the slope (or gradient) of linear interpolation of the received signal strength in respect to the $10 \log (d)$ :

$$
P_{\mathrm{RX}}=P_{\mathrm{TX}}-\alpha 10 \log (d)+N
$$




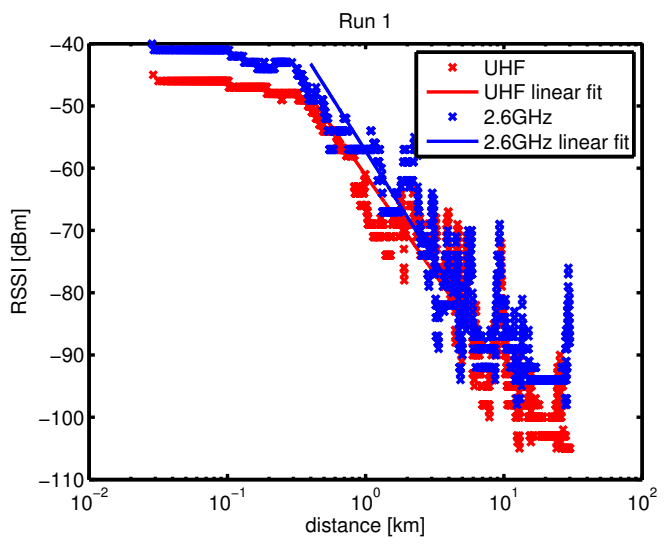

Fig. 8. Path loss component

As an example we plot the results from trial 2, run 1 in Figure 8 . The average estimated path loss component for the $800 \mathrm{MHz}$ band is 3.2 and for $2.6 \mathrm{GHz}$ is 3.5 , which is in line with established path loss models for rural areas.

\section{B. Delay and Doppler Spectra}

We first show the results for the $800 \mathrm{MHz}$ band. In Figure 9 we show the Delay-Doppler Power Spectrum $\mathbf{S}_{t, u, k}$ of trial 1, run 1 for three different blocks. At $t=50$ the train is approaching the base station, at $t=90$ it is the closest to the base station and at $t=130$ it is departing from the base station. It can be seen that there is one dominant component in the spectrum corresponding to the line of sight (LOS), which is moving from approximately $f_{1}=-625 \mathrm{~Hz}$ to $f_{2}=$ $-1040 \mathrm{~Hz}$. This effect can be seen even better in Figure 10, where we plot the marginal Doppler Profile $\mathbf{D}_{t, u}$ over the whole run. The difference between these two frequencies correspond more or less exactly to Doppler bandwidth $B_{D}=2 f_{c} \frac{v_{\max }}{c} \approx f_{2}-f_{1}$. The common offset $f_{o}=\frac{f_{1}+f_{2}}{2}$ correspond to the frequency offset in the system, which was (unfortunately) not calibrated beforehand in the first trial.

For the $2.6 \mathrm{GHz}$ band we show the Delay-Doppler Power Spectrum $\mathbf{S}_{t, u, k}$ of trial 1, run 1, carrier $\mathrm{A}$ $(10 \mathrm{MHz})$ in Figure 11 for three different blocks (approaching, close, departing). Moreover, we plot the temporal evolution of the marginal Doppler profile in Figure 12. Compared to the measurement in the $800 \mathrm{MHz}$ band, we can see that the Doppler component at $f 1=1040 \mathrm{~Hz}$ persists after the train passes the base station in addition to the second Doppler component appearing at $f 2=$ $-370 \mathrm{~Hz}$. Our explanation for this behavior is that there must be a strong reflector directly on the train somewhere between the front of the train and the antenna. Indeed, the IRIS 320 train has two "observation towers" at the front and at the rear with a large glass surface acting as a reflector. In the measurements we can only see the tower

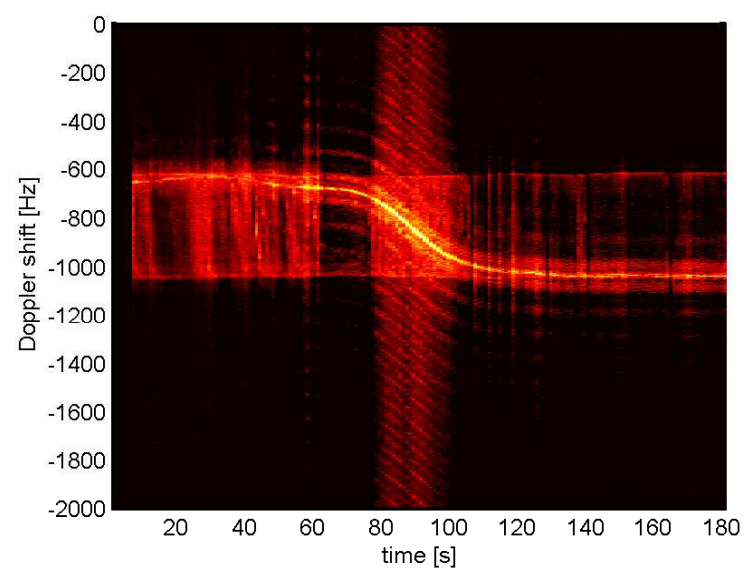

Fig. 10. Doppler Profile for the $800 \mathrm{MHz}$ band, trial 1 , run 1

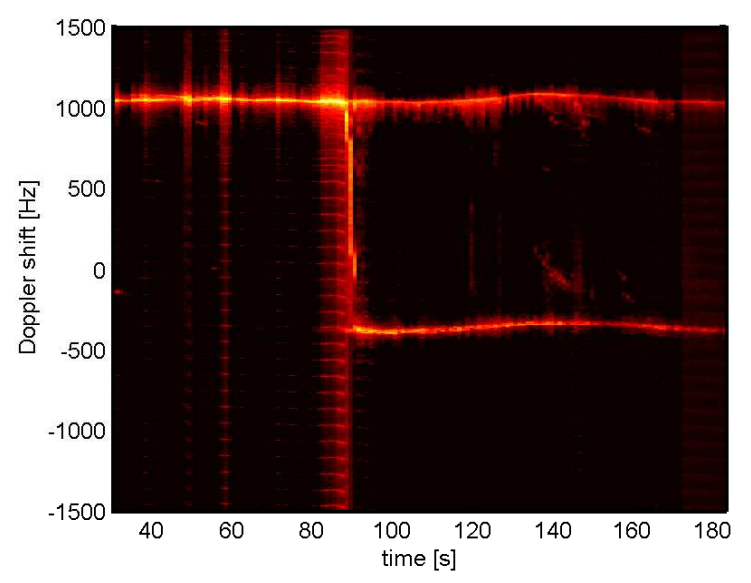

Fig. 12. Doppler Profile for the 2.6GHz band, trial 2, run 1

in the front, since the antenna is directional and pointing only to the front. This hypothesis can be confirmed by looking at the results of run 2 , where the train takes the same route in the other direction. As can be seen in Figure 13, here the two Doppler components are present when the train approaches the base station and vanish when the train has passed the base station. Moreover this phenomenon can be observed on both carriers at $2.6 \mathrm{GHz}$ (not shown). It is however interesting that this phenomenon does not exist in the $800 \mathrm{MHz}$ band, which can be explained by the fact that this antenna has a higher attenuation along the horizontal plane. It could also be that the reflector material has different reflection coefficients in the $800 \mathrm{MHz}$ band.

\section{CONCLUSIONS}

Achieving broadband wireless communication for high speed trains is not trivial and requires a good understanding of the underlying wireless communica- 

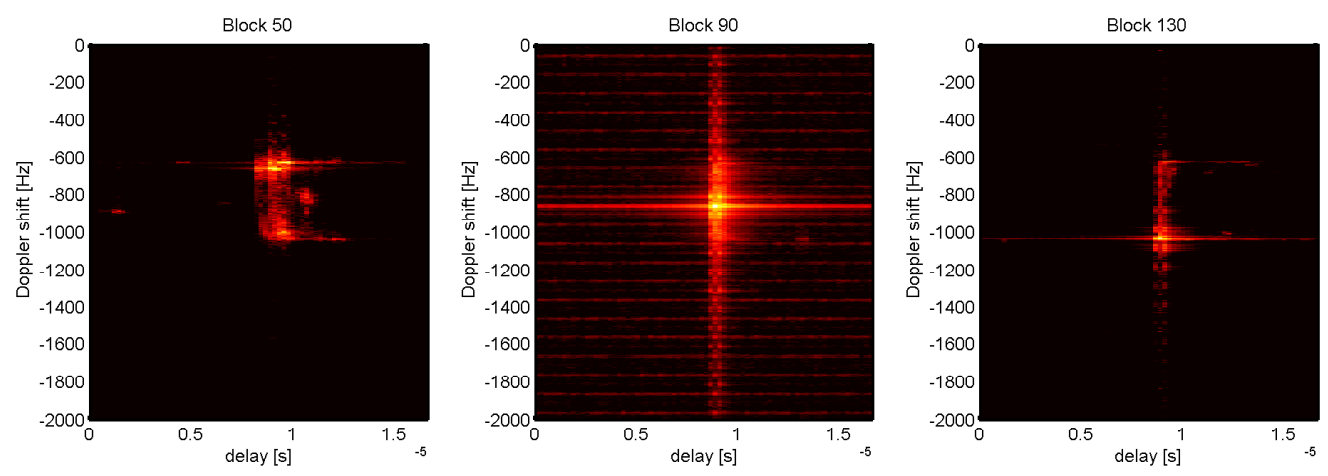

Fig. 9. Doppler Delay Power Spectrum for the $800 \mathrm{MHz}$ band, trial 1 , run 1
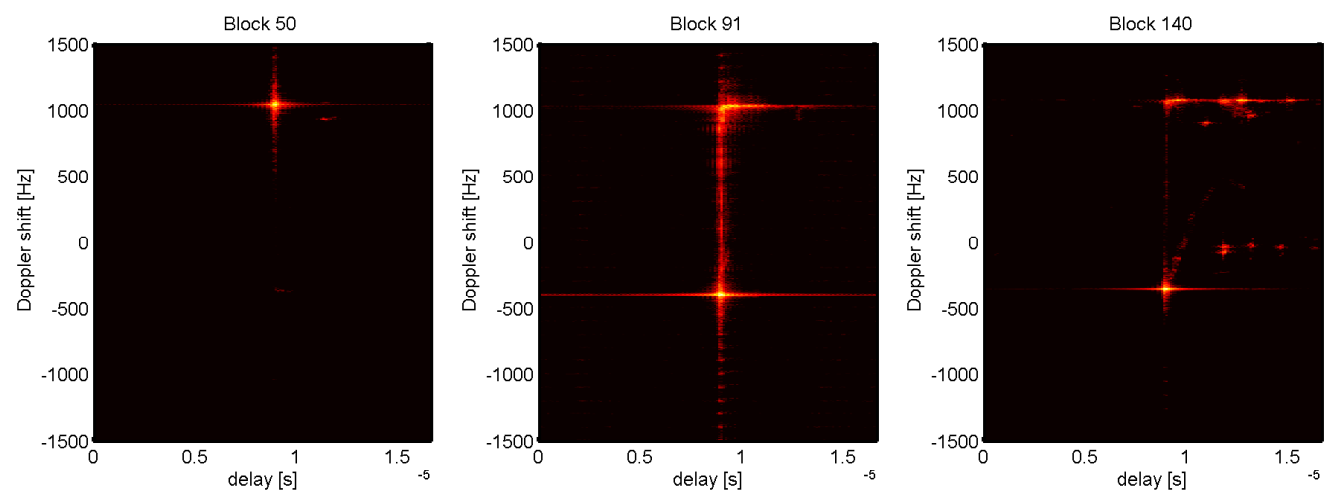

Fig. 11. Doppler Delay Power Spectrum for the $2.6 \mathrm{GHz}$ band, trial 2, run 1

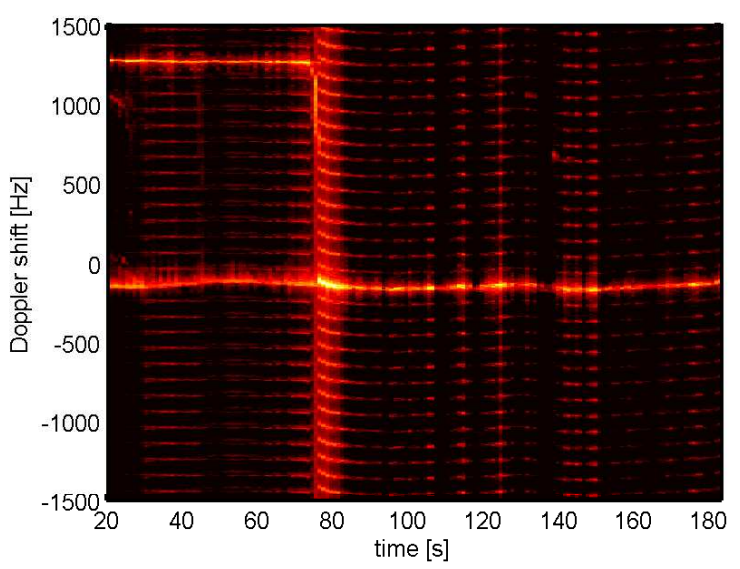

Fig. 13. Doppler Profile for the $2.6 \mathrm{GHz}$ band, trial 2, run 2

tion channel. We have presented a channel sounding measurement campaign carried out in the context of the project CORRIDOR and presented some initial results. A surprising result was the reflection that comes from the observation tower of the IRIS 320 train and which results in a very large Doppler spread that can have a negative impact on the communication link as it results in high

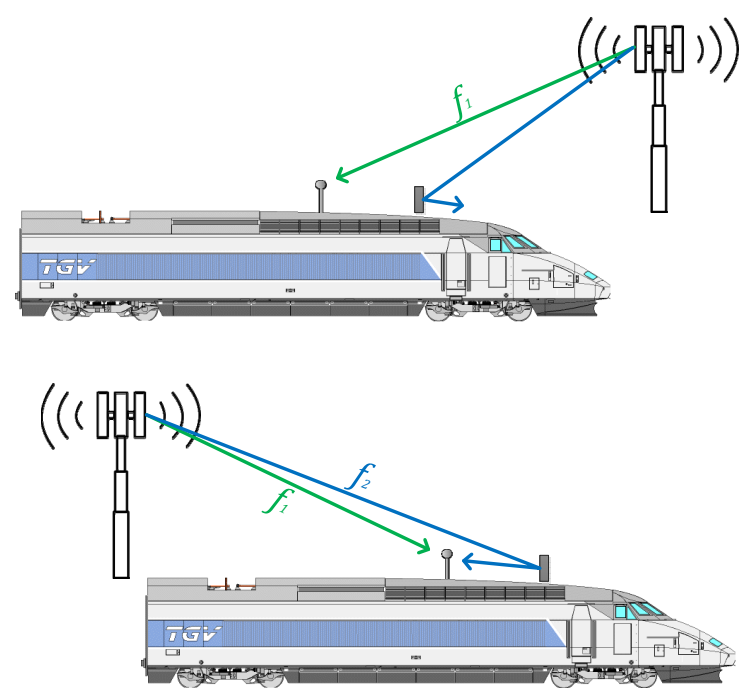

Fig. 14. Explanation of the Doppler Profile. Top: only one strong Doppler component is present. Bottom: due to a reflector in the front of the train two Doppler components with opposite signs are present. 
inter-carrier interference. However, this effect should not be present on a regular train. In future work we will analyze the spatial properties of the measured channels and fit suitable channel models to the data.

\section{ACKNOWLEDGEMENTS}

This work has been supported by the projects CORRIDOR (ANR), SOLDER (EU FP7), and SHARING (Celtic+). The authors would also like to thank the CNES for providing the $800 \mathrm{MHz}$ power amplifiers as well as Claude Oestges for his advice.

\section{REFERENCES}

[1] Z. Min, W. Muqing, S. Yanzhi, Y. Deshui, D. Shiping, Z. Panfeng, Z. Xiangbing, and G. Shuyun, "Analysis and modeling for trainground wireless wideband channel of lte on high-speed railway," in Vehicular Technology Conference (VTC Spring), 2013 IEEE 77th, June 2013, pp. 1-5.

[2] Z. Min, W. Muqing, S. Yanzhi, J. Guiyuan, D. Shiping, Z. Panfeng, Z. Xiangbing, and G. Shuyun, "Analysis and modeling of the lte broadband channel for train-ground communications on highspeed railway," in Vehicular Technology Conference (VTC Fall), 2013 IEEE 78th, Sept 2013, pp. 1-5.

[3] A. Paier, J. Karedal, N. Czink, H. Hofstetter, C. Dumard, T. Zemen, and C. F. Mecklenbrauker, "Car-to-car radio channel measurements at $5 \mathrm{ghz}$ : Pathloss, power-delay profile, and delaydoppler spectrum," in International Symposium on Wireless Communication Systems (ISWCS 2007), Oct. 2007, pp. 224-228. 